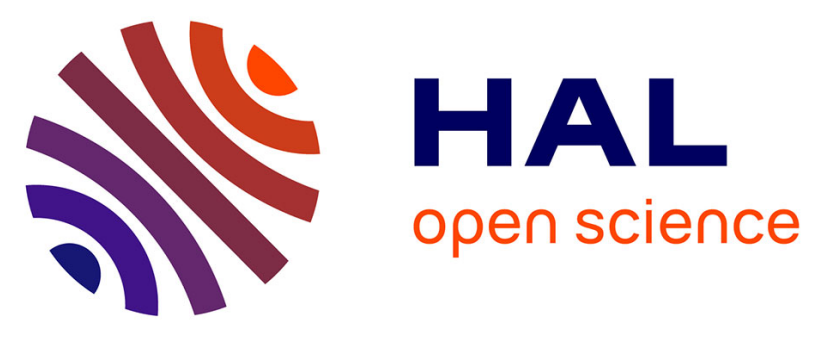

\title{
Studying One and Two-Finger Perception of Tactile Directional Cues
}

Yoren Gaffary, Maud Marchal, Adrien Girard, Marine Pellan, Anouk Asselin, Benoît Peigné, Mathieu Emily, Florian Gosselin, Anthony Chabrier, Anatole Lécuyer

\section{To cite this version:}

Yoren Gaffary, Maud Marchal, Adrien Girard, Marine Pellan, Anouk Asselin, et al.. Studying One and Two-Finger Perception of Tactile Directional Cues. 10th International Conference on Haptics Perception, Devices, Control, and Applications (EuroHaptics), Imperial College London, Jul 2016, Londres, United Kingdom. pp.396-405, 10.1007/978-3-319-42324-1_39 . hal-01406434

\section{HAL Id: hal-01406434 https://hal.science/hal-01406434}

Submitted on 12 Dec 2016

HAL is a multi-disciplinary open access archive for the deposit and dissemination of scientific research documents, whether they are published or not. The documents may come from teaching and research institutions in France or abroad, or from public or private research centers.
L'archive ouverte pluridisciplinaire HAL, est destinée au dépôt et à la diffusion de documents scientifiques de niveau recherche, publiés ou non, émanant des établissements d'enseignement et de recherche français ou étrangers, des laboratoires publics ou privés. 


\title{
Studying one and two-finger perception of tactile directional cues
}

\author{
Yoren Gaffary $^{1}$, Maud Marchal ${ }^{1,2}$, Adrien Girard ${ }^{1}$, Marine Pellan $^{3}$, Anouk \\ Asselin $^{3}$, Benoit Peigne ${ }^{3}$, Mathieu Emily ${ }^{3,4}$, Florian Gosselin ${ }^{5}$, Anthony \\ Chabrier $^{5}$, Anatole Lécuyer ${ }^{1,6}$ \\ 1 Inria, Campus de Beaulieu, 35042 Rennes, France \\ 2 INSA, 20 Avenue des Buttes de Coesmes, 35708 Rennes, France \\ 3 Agrocampus Ouest, 65, rue de Saint Brieuc, 35042 Rennes Cedex, France \\ 4 Agrocampus Ouest - IRMAR UMR CNRS 6625, 35042 Rennes Cedex, France \\ 5 CEA, LIST, Interactive Robotics Laboratory, F-91190 Gif-sur-Yvette, France \\ 6 IRISA, 263 Avenue Général Leclerc, 35000 Rennes
}

\begin{abstract}
In this paper, we study the perception of tactile directional cues by one or two fingers, using either the index, middle, or ring finger, or any of their combination. Therefore, we use tactile devices able to stretch the skin of the fingertips in 2 DOF along four directions: horizontal, vertical, and the two diagonals. We measure the recognition rate in each direction, as well as the subjective preference, depending on the (couple of) finger(s) stimulated. Our results show first that using the index and/or middle finger performs significantly better than using the ring finger on both qualitative and quantitative measures. The results when comparing one versus two-finger configurations are more contrasted. The recognition rate of the diagonals is higher when using one finger than two, whereas two fingers enable a better perception of the horizontal direction. These results pave the way to other studies on one versus two-finger perception, and raise methodological considerations for the design of multi-finger tactile devices.
\end{abstract}

Keywords: tactile perception, fingertip stimulation, multifinger

\section{Introduction}

The fingertips are among the most sensitive parts of the human body 9 . When haptically exploring the world with our hands, we usually make use of multiple fingers and multiple fingertips. In doing so, it is commonly considered that the more fingers involved the better perception, and that, among fingers the index fingertip is the most sensitive and efficient one. But there are actually rather few studies which specifically compare the perception of tactile cues using the index versus the other fingers and/or a single-finger versus multiple fingers.

Numerous haptic studies investigated the tactile perception obtained with one finger at the level of fingertip [1015]. As an example, Gleeson et al. 4] studied the perception of a tactile directional cue applied on a single finger. They 
designed a tactile display enabling skin displacement at the level of fingertip and evaluated the perception of two different directions of stimulation along the horizontal axis (left, right) with the right index.

On the contrary, only few studies investigated the use of several fingers ([6/7]). For example, a game controller able to stimulate both thumbs using a tactor displacement was proposed by Guinan et al. [6]. This setup involved stimulating both thumbs of the user with four possible directional stimuli. The stimuli were displayed sequentially on both thumbs. Recognition rate depended on the delay time between the two displays. Jansson and Monaci 7 investigated the influence of the number of fingers used on the tactile exploration of a 2D tactile map. They did not find a clear improvement when using two fingers instead of one. However, they observed a significant learning effect when using two fingers, and an improvement of performance over sessions in this case.

In this paper we specifically address the influence of the finger used (index, middle, ring), or the possibility to use two fingers simultaneously, for the perception of tactile directional cues. We present an experiment in which directional stimuli are displayed either on the index, middle or ring finger, or on any couple of these fingers. We measure both the recognition rate in four directions (horizontal, vertical, and the two diagonals), and the subjective preference of participants.

The next section describes our experimental method for studying tactile perception using one or two fingers. We successively present our experimental apparatus based on a 2 DOF tactile display, our experimental protocol, and the data we collected. The section 3 presents the results of this experiment, followed by a conclusion.

\section{User study: One and two-finger perception of tactile directional cues}

\subsection{Objective and Hypotheses}

Our study aims at determining which combinations of index, middle or ring finger allow the most accurate perception, i.e., the highest recognition rate regarding the direction of the haptic stimuli displayed to the participants (vertical, horizontal, diagonal). In this study, we specifically focused our attention on a combination between one and two fingers.

Three hypotheses are evaluated:

H1 Finger efficiency. The accurate perception of a stimulus relies on the finger(s) used to perceive this stimulus. Commonly, the index is thought as the most accurate to perceive directions [5].

H2 Redundancy efficiency. The redundant display of a stimulus on two fingers provides a higher recognition rate than if presented on one finger alone, as more cutaneous receptors are stimulated.

H3 Learning effect. The recognition rate increases during the experiment due to the presence of a learning effect when using two fingers as suggested in [7. 


\subsection{Experimental setup}

The experimental setup is based on a tactile device called Haptip and presented in [3. (see Figure 1a). It stimulates fingertips through the 2D displacement of a tactor. This kind of skin stretching device is suitable to display directional information on the fingertips [4].

The experiment includes either one or two devices depending on the number of fingers to be stimulated (see Figures $1 \mathrm{~b}$ and $1 \mathrm{c}$ ). When a single finger is stimulated, only one device is required. Two identical devices are required when two fingers are stimulated. In this case, the two devices are synchronized using the API provided by the manufacturer to display the same stimulus at the same time.

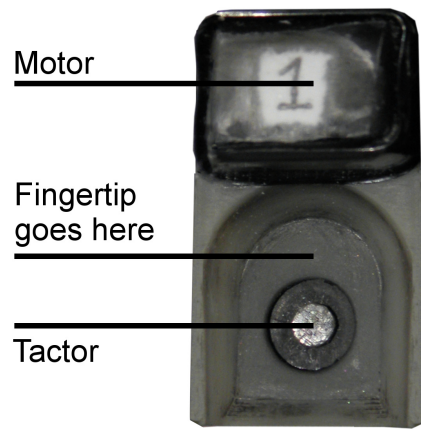

(a) Tactor displacement based tactile device.

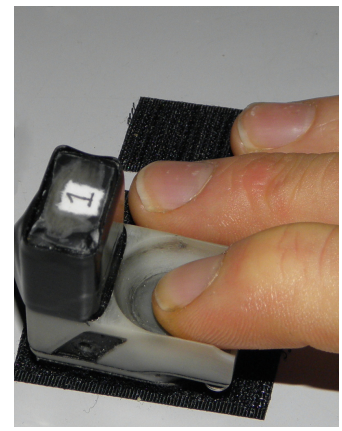

(b) Haptic perception with the index (one actuator).

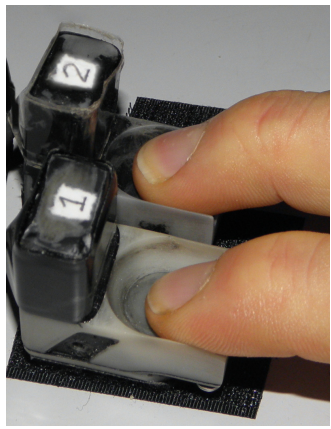

(c) Haptic perception with index-middle (two actuators).

Fig. 1: Tactile device used in the study: (a) top view of the device, (b) and (c) profile views with one or two actuated tactors.

\subsection{Experimental plan}

Apparatus. The participants were seated with their forearms placed on a table. The participants were seated in front of a 24 inch screen displaying the questions concerning the stimuli directions, with their forearms placed on a table (see Fig. 2). The haptic devices were fixed to the table in front of the dominant hand of the participant using velcro. The velcro was used to prevent the devices from moving during the experiment. The position of each tactor on the table was adjusted to the length of the participant's finger. The non-dominant hand of the participant was holding a mouse used to answer the questions displayed on the screen. 


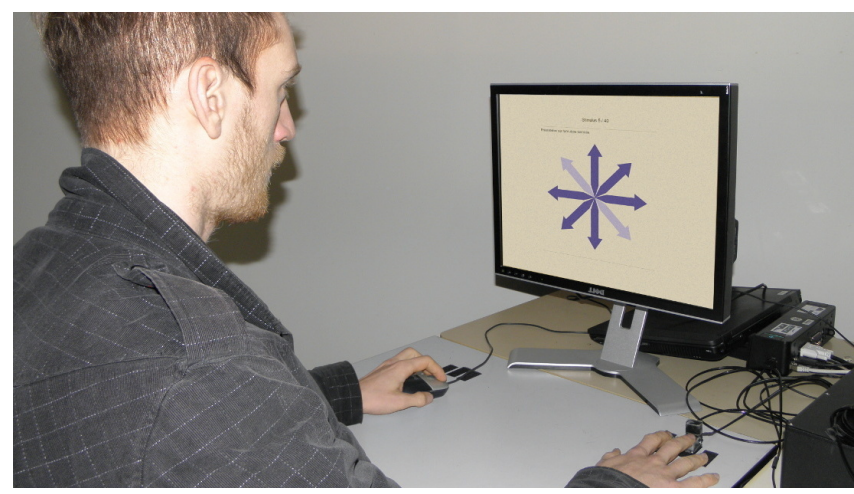

Fig. 2: Experimental apparatus: example of a participant reporting the direction of the stimulus he just perceived on the fingertip of his middle finger (here $45^{\circ}$ ).

Participants. Eighteen participants took part to the experiment. There were 7 males and 11 females, aged from 21 to 32 (mean=23, SD =2.8). 15 participants identified their right hand as the dominant one. When asked about their experience with haptics, 10 participants reported that they were familiar, 8 reported that they were not familiar.

Procedure. Participants were asked to complete a perception task that consisted in recognizing the direction of the tactile stimuli that were displayed under their fingers. The different possible directions were displayed on the screen and each participant could answer using a mouse in his or her non-dominant hand. At the beginning of the experiment, the instructor presented the different tactile stimuli and explained them how to put their fingers on the device. Each participant completed a short training session during which a random stimulus was presented to him.

Four different stimuli were presented to the participants, each corresponding to a specific direction in space. These stimuli were generated through specific displacements of the tactor scratching the fingertip. All the stimuli used the maximum range of motion of the device (i.e. $4 \mathrm{~mm}$ ) and lasted one second. The stimuli differed by the direction of their movement from the vertical: $0^{\circ}, 45^{\circ}, 90^{\circ}$ and $135^{\circ}$ in direct order. The displacement of each tactor followed a sinusoidal law, with an amplitude of $4 \mathrm{~mm}$ and a frequency of $1 \mathrm{~Hz}$. For each stimulus, the tactor starts from the center of the device, and then moves in three steps: 1) the tactor moves to an extremity, 2) the tactor moves to the opposite extremity, crossing the center, and 3) the tactor moves to the center. Figure 3 illustrates the four directions with the three different steps of the tactor movement.

The stimuli were presented to the participants on a combination of one or two fingers, the possible combinations being: the index, middle or ring finger alone, and index-middle, index-ring and middle-ring couples. The thumb was 


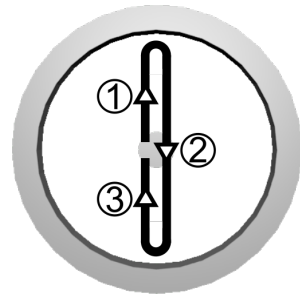

(a) Vert. $=0^{\circ}$

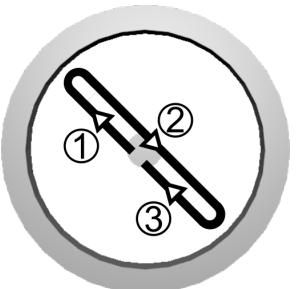

(b) Diag. $1=45^{\circ}$

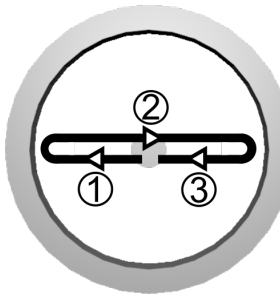

(c) Hori. $=90^{\circ}$

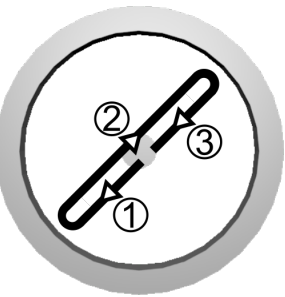

(d) Diag. $2=135^{\circ}$

Fig. 3: The four different stimuli displayed to the participants. The tactor displacement follows four possible directions: vertical, horizontal or one of the two diagonals.

not considered in this study as the stimuli could not be presented in the same configuration as for the other fingers. The little finger was also removed as our haptic device was too large for this finger.

All 4 directions $\times 6$ fingers combinations were presented to each participant. A trial was considered valid after 1 s stimulus and when the participant had reported a direction between the four proposed on the screen using the mouse in his or her non-dominant hand. The participant could ask for a break at any time between each trial.

Conditions and experimental design. The independent variables of the experiment were: the Finger used to perceive the stimulus (INDEX, MidDLE, Ring, Index-Middle, Index-Ring and Middle-Ring), the Direction of the stimuli displayed on the fingertips ( Vertical, Horizontal, Diagonal 1 and Diagonal 2) and REPETITION (10 different trials for each finger combination and each direction). While the directions of the stimuli were randomly presented, the order of the fingers used to perceived the stimuli was counterbalanced across participants to avoid privileging a combination of fingers among another one. The duration of the experiment was approximately 30 minutes including breaks. In total, we had 18 participants $\times 6$ finger combinations $\times 4$ directions $\times 10$ repetitions $=4,320$ trials.

\section{Collected data.}

Main measure. We recorded the perceived direction of the stimulus presented in each case. Therefore, the participants had to select the direction of the stimulus they perceived between four possible directions using arrows (see Fig. 2). This measure aims at evaluating the influence of the finger on recognition rate, redundancy efficiency and learning effect $(\mathbf{H 1}, \mathbf{H 2}$ and $\mathbf{H 3})$. 
Subjective questionnaire. At the end of the experiment, the participant fulfilled a subjective questionnaire to evaluate the coherence between their objective performances and their subjective feeling.

The two first questions were:

Ms1 "Was it is easy to recognize directions with the $X$ finger(s)?" This question was asked six times, one for each combination of fingers. Participants answered each time using a 5-item Likert scale.

Ms2 "Classify the directions by their easiness to recognize". There were three items to order: horizontal stimuli, vertical stimuli and diagonal stimuli.

Following questions were answered by participants using a 5-item Likert scale:

Ms3 "Did you feel the perception was easier with a combination of two fingers instead of one finger?" This question aims at evaluating the coherence between perceived redundancy efficiency and true redundancy efficiency.

Ms4 "Did your perception changed during the experiment?" This question aims at evaluating the subjective perception of a learning effect.

Ms5 "Was the task easy to perform?" This question aims at determining if the selected stimuli were not too easy or too difficult to recognize.

\section{Results and Analysis}

\subsection{Recognition rate}

To study the recognition rate of the direction of the stimuli in function of the combination of fingers, we used a logistic regression model on the collected data to model the probability of recognition with respect to the independent variables Finger and DiRection defined in the experimental design. The probability is thus comprised between 0 and $1,0.25$ corresponding to the guess rate, a higher probability highlighting a better recognition rate while a lower probability highlighting the confusions between the potential answers. The participants are considered as a random effect in the model.

Learning effect. In order to investigate the presence of a learning effect during the experiment, we incorporated the REPETITION factor in the model. We separated the $i$ first presented stimuli for each combination of fingers from the $40-i$ last stimuli, with $i \in[1,39]$. Results showed that for $i \in[1,13]$, the first stimuli provided lower recognition rate $(74 \%$ vs $77 \%$ correct recognition rate, $\mathrm{p}$-values $\in[0.004,0.047])$. These results highlight a learning effect and support H3. The statistical analysis reported in the following section were then performed by removing the first 13 stimuli from the data of each combination of finger and for each participant. 
Main analysis. We performed an analysis of deviance of the logistic regression model and we found a significant marginal effect for both Finger $(p=0.003)$ and Direction $(p<0.001)$, as well as an interaction effect between Finger and Direction $(p=0.04)$. Table 1 displays the recognition rates in function of the displayed directions and combination of fingers.

We performed a post-hoc analysis on the Direction using a Tukey test adapted to the logistic generalized regression model. We found that the VERTICAL direction was significantly better recognized than the other directions $(Z=-6.56, Z=-5.25, Z=-4.34$ for the Diagonal 1 , Horizontal and DiAGONAL 2 and $p<0.001$ for the three directions). We did not find a significant effect between the other directions.

We performed a post-hoc analysis on the FINGER using the same Tukey test. We found that the stimuli display on the INDEX finger were significantly better recognized than the MidDLE-RING combination $(Z=3.2, p=0.18)$. We also found that the stimuli displayed on the INDEX-MIDDLE finger were significantly better recognized than the MidDLE-RING combination $(Z=3.5, p=0.005)$. We did not find any other significant effect between the other combinations of fingers.

Interaction between DiRECTION and Finger. We performed a post-hoc analysis to analyze the interaction between the DireCtion and Finger variables using a pairwise comparison based on least squares means estimates. All the significant differences concerned the VERTICAL direction. We found that the two diagonal directions Diagonal 1 and Diagonal 2 for the Middle-Ring combination were less recognized than the VERTICAL direction for the following finger combinations: INDEX $(Z=4.59, p<0.01$ and $Z=4.41, p<0.01$ respectively), MidDle $(Z=4.75, p<0.01$ and $Z=4.58, p<0.01$ respectively), INDEX-MiddLE ( $Z=4.6, p<0.01$ and $Z=4.42, p<0.01$ respectively), INDEX-RING $(Z=3.88, p=0.02$ and $Z=3.69, p=0.04$ respectively). We found also that the VERTICAL direction for the combinations MidDLE alone and INDEX-MIDDLE had a significantly higher recognition rate than the HORIZONTAL direction for the INDEX and MidDLE fingers $(Z=3.83, p=0.02 ; Z=3.88$, $p=0.02 ; Z=3.63, p=0.04$ and $Z=3.69, p=0.04$ respectively). Finally, we found that the VERTICAL direction for the INDEX was better recognized than the HORIZONTAL direction for the MIDDLE finger $(Z=3.68, p=0.04)$, and that the VERTICAL direction for the MidDle was better recognized than the Diagonal 1 direction for the INDEX-RING combination $(Z=3.78, p=0.02)$.

One vs. two fingers. To investigate the influence of the number of fingers used to perceive the stimuli on recognition rate, we grouped together the results obtained with the same number of fingers (the variable FINGER has only two values: one vs. two fingers). As previously, we used a logistic regression model on the collected data to model the probability of recognition with respect to the independent variables Finger and DiREction. We performed an analysis of deviance of the logistic regression model and we found a significant marginal effect for Direction $(p<0.001)$, as well as an interaction effect between Finger 
Table 1: Recognition rate (in percent) according to the direction of the stimuli and the fingers used to perceived them. The grey cells represent the cells where the perceived direction is the direction of the stimulus.

\begin{tabular}{|c|c|c|c|c|c|}
\hline Orientation & \multicolumn{5}{|c|}{ Stimulus direction } \\
\hline \multirow{5}{*}{$\begin{array}{l}\text { Index } \\
\text { finger }\end{array}$} & & $0^{\circ}$ & $45^{\circ}$ & $90^{\circ}$ & $135^{\circ}$ \\
\hline & $0^{\circ}$ & $86.7 \%$ & $8.9 \%$ & $0.0 \%$ & $10.0 \%$ \\
\hline & $45^{\circ}$ & $9.4 \%$ & $75.6 \%$ & $23.9 \%$ & $1.7 \%$ \\
\hline & $90^{\circ}$ & $0.6 \%$ & $15.6 \%$ & $70.6 \%$ & $9.4 \%$ \\
\hline & $135^{\circ}$ & $3.3 \%$ & $0.0 \%$ & $5.6 \%$ & $78.9 \%$ \\
\hline & \multicolumn{5}{|c|}{ Stimulus direction } \\
\hline \multirow{5}{*}{$\begin{array}{l}\text { Middle } \\
\text { finger }\end{array}$} & & $0^{\circ}$ & $45^{\circ}$ & $90^{\circ}$ & $135^{\circ}$ \\
\hline & $0^{\circ}$ & $87.8 \%$ & $8.9 \%$ & $0.0 \%$ & $3.9 \%$ \\
\hline & $45^{\circ}$ & $8.3 \%$ & $76.7 \%$ & $17.8 \%$ & $3.9 \%$ \\
\hline & $90^{\circ}$ & $0.0 \%$ & $14.4 \%$ & $70.6 \%$ & $12.2 \%$ \\
\hline & $135^{\circ}$ & $3.9 \%$ & $0.0 \%$ & $11.7 \%$ & $80.0 \%$ \\
\hline & \multicolumn{5}{|c|}{ Stimulus direction } \\
\hline \multirow{6}{*}{$\begin{array}{l}\text { Ring } \\
\text { finger }\end{array}$} & & $0^{\circ}$ & $45^{\circ}$ & $90^{\circ}$ & $135^{\circ}$ \\
\hline & $0^{\circ}$ & $84.4 \%$ & $6.1 \%$ & $1.1 \%$ & $12.8 \%$ \\
\hline & $45^{\circ}$ & $13.9 \%$ & $71.1 \%$ & $19.4 \%$ & $2.8 \%$ \\
\hline & $90^{\circ}$ & $0.6 \%$ & $20.6 \%$ & $73.3 \%$ & $14.4 \%$ \\
\hline & $135^{\circ}$ & $1.1 \%$ & $2.2 \%$ & $6.1 \%$ & $70.0 \%$ \\
\hline & \multicolumn{5}{|c|}{ Stimulus direction } \\
\hline \multirow{6}{*}{$\begin{array}{l}\text { Index-middle } \\
\text { fingers }\end{array}$} & & $0^{\circ}$ & $45^{\circ}$ & $90^{\circ}$ & $135^{\circ}$ \\
\hline & $0^{\circ}$ & $87.2 \%$ & $10.0 \%$ & $0.6 \%$ & $5.6 \%$ \\
\hline & $45^{\circ}$ & $6.7 \%$ & $71.1 \%$ & $16.7 \%$ & $1.7 \%$ \\
\hline & $90^{\circ}$ & $0.6 \%$ & $17.8 \%$ & $77.8 \%$ & $15.0 \%$ \\
\hline & $135^{\circ}$ & $5.6 \%$ & $1.1 \%$ & $5.0 \%$ & $77.8 \%$ \\
\hline & \multicolumn{5}{|c|}{ Stimulus direction } \\
\hline \multirow{5}{*}{$\begin{array}{l}\text { Index-ring } \\
\text { fingers }\end{array}$} & & $0^{\circ}$ & $45^{\circ}$ & $90^{\circ}$ & $135^{\circ}$ \\
\hline & $0^{\circ}$ & $83.9 \%$ & $4.4 \%$ & $0.0 \%$ & $6.1 \%$ \\
\hline & $45^{\circ}$ & $14.4 \%$ & $68.9 \%$ & $15.6 \%$ & $1.7 \%$ \\
\hline & $90^{\circ}$ & $0.0 \%$ & $25.6 \%$ & $78.3 \%$ & $18.3 \%$ \\
\hline & $135^{\circ}$ & $1.7 \%$ & $1.1 \%$ & $6.1 \%$ & $73.9 \%$ \\
\hline & \multicolumn{5}{|c|}{ Stimulus direction } \\
\hline \multirow{5}{*}{$\begin{array}{l}\text { Middle-ring } \\
\text { fingers }\end{array}$} & & $0^{\circ}$ & $45^{\circ}$ & $90^{\circ}$ & $135^{\circ}$ \\
\hline & $0^{\circ}$ & $74.4 \%$ & $10.0 \%$ & $0.6 \%$ & $15.6 \%$ \\
\hline & $45^{\circ}$ & $19.4 \%$ & $62.2 \%$ & $16.1 \%$ & $4.4 \%$ \\
\hline & $90^{\circ}$ & $1.1 \%$ & $25.6 \%$ & $78.9 \%$ & $11.7 \%$ \\
\hline & $135^{\circ}$ & $5.0 \%$ & $2.2 \%$ & $4.4 \%$ & $68.3 \%$ \\
\hline
\end{tabular}

and Direction $(p=0.04)$. We did not find any significant effect this time for the FINGER combination.

Concerning the DiREction, we performed a post-hoc analysis using a Tukey test. We found the same significant effects: the VERTICAL direction was significantly better recognized than the others directions $(Z=-6.44, Z=-5.1$, $Z=-4.4$ for the Diagonal 1 , Horizontal and Diagonal 2 and $p<0.001$ for the three directions). We did not find a significant effect between the other directions.

Concerning the interaction, we found that the VERTICAL direction for ONE FINGER was better recognized than the other combinations, except for the VERTICAL direction for TWO FINGERs. We also found that the DiAgONAL 1 direction for Two FINGERs was less recognized than the HorizonTAL direction for Two 
Fingers $(Z=-3.22, p=0.03)$ and the DiAGONAL 2 direction for ONE FINGER $(Z=-3.25, p=0.02)$.

\subsection{Subjective questionnaire}

The results of our subjective questionnaires showed that the condition allowing to recognize the directions the most easily was MidDLE (Ms1, $\mathrm{M}=3.67 / 5)$. At the opposite, the RING alone was perceived as the most difficult condition to recognize stimuli $(\mathrm{M}=3.11 / 5)$. Participants reported that the Vertical direction was the easiest to recognize (Ms2, for $77.8 \%$ of the participants). At the opposite, $88.9 \%$ of the participants reported that the Diagonal direction was the most difficult to recognize. Concerning the subjective evaluation of one vs two finger configurations (Ms3), participants reported they did not feel any difference between one and two-finger display presentation $(\mathrm{M}=3.11 / 5)$. Participants reported they felt an evolution of their tactile performance during the experiment (Ms4, for $77 \%$ of the participants). More specifically, the participants reported that their performance increased during the experiment (median: 4). This is in line with the objective results, especially for two-finger configurations. Finally, the participants reported the task was not too easy or too difficult (Ms5, M = 3.11/5).

\subsection{Results summary}

For one finger configurations, we observed a higher recognition rate with the INDEX and MIDDLE than with the RING finger, in accordance with 815. We also observed that the VERTICAL direction displays the highest recognition accuracy. This is inline with 112 as this direction is generally better recognized as a proximodistal axis.

Regarding two fingers configurations, we observed a learning effect as previously mentioned in [7. We also found that the two diagonal directions DiaGONAL 1 and Diagonal 2 for the Middle-Ring combination were less recognized than the VERTICAL direction for the other combinations. This explains the low results for the MidDLe-Ring.

Finally, our results highlight a difference between one and two-fingers configurations. Two-fingers combinations seem to be more effective for perceiving the HORIZONTAL direction. However, diagonals seem to be more commonly mistaken with the HORIZONTAL direction in a two-finger configuration.

The subjective appreciation of the participants (obtained with the subjective questionnaire) is consistent with their objective accuracy.

\section{Conclusion}

This study aimed at determining the influence of the number of fingers used to perceive directional stimuli on recognition accuracy. Three fingers were involved: index, middle and ring. 
The results of our experiment showed that the fingers used and their number influence the accuracy in perceiving directions. Among single fingers, the index and middle gave the highest recognition rate. The influence of the number of fingers displayed contrasted results. The diagonals are better perceived using a single finger, but the horizontal direction is better perceived using two fingers.

Future works could investigate the influence of parameters such as the position of the fingers during the stimulation or distance between fingers. This would enable to further investigate the relation between the number of fingers used and the perceived directions. Different durations and shapes of stimuli could also be tested. In a long-term perspective, these results could serve as guidelines for the development of a new haptic device aiming at stimulating effectively fingertips.

\section{Acknowledgments}

This work was supported by the European Commission through the HAPPINESS project (SEP-210153552) and by French National Research Agency through the MANDARIN project (ANR-12-CORD-0011) labeled by French Cluster Cap Digital.

\section{References}

1. Essock, E.a., Krebs, W.K., Prather, J.R.: Superior sensitivity for tactile stimuli oriented proximally-distally on the finger: Implications for mixed class 1 and class 2 anisotropies. Journal of Experimental Psychology: Human Perception and Performance 23(2), 515-527 (1997)

2. Gentaz, E., Ballaz, C.: The visual perception of orientation, and "the oblique effect". Annee Psychologique 100(4), 715-744 (2000)

3. Girard, A., Marchal, M., Gosselin, F., Chabrier, A., Louveau, F., Lécuyer, A.: HapTip: Displaying Haptic Shear Forces at the Fingertips for Multi-Finger Interaction in Virtual Environments. Frontiers in ICT (2016)

4. Gleeson, B., Horschel, S., Provancher, W.: Design of a Fingertip-Mounted Tactile Display with Tangential Skin Displacement Feedback. IEEE Transactions on Haptics 3(4), 297-301 (2010)

5. Gleeson, B.T., Horschel, S.K., Provancher, W.R.: Perception of Direction for Applied Tangential Skin Displacement: Effects of Speed, Displacement, and Repetition. IEEE Transactions on Haptics 3(3), 177-188 (2010)

6. Guinan, A.L., Caswell, N.A., Drews, F.A., Provancher, W.R.: A video game controller with skin stretch haptic feedback. Digest of Technical Papers - IEEE International Conference on Consumer Electronics pp. 456-457 (2013)

7. Jansson, G., Monaci, L.: Exploring tactile maps with one or two fingers. Cartographic journal 40(3), 269-271 (2003)

8. King, H.H., Donlin, R., Hannaford, B.: Perceptual thresholds for single vs. multifinger haptic interaction. In: IEEE Haptics Symposium. pp. 95-99. Waltham, MA (2010)

9. Scheibe, R., Moehring, M., Froehlich, B.: Tactile feedback at the finger tips for improved direct interaction in immersive environments. IEEE Symposium on 3D User Interfaces pp. 123-130 (2007)

10. Vitello, M., Ernst, M., Fritschi, M.: An instance of tactile suppression: Active exploration impairs tactile sensitivity for the direction of lateral movement. Proceedings of the 2006 EuroHaptics Conference pp. 351-355 (2006) 